\title{
Temporal Trends in Peripheral Arterial Interventions: Observations from the Blue Cross Blue Shield of Michigan Cardiovascular Consortium (BMC2 PVI)
}

\author{
Short Title: Trends in Peripheral Arterial Interventions
}

Michael P. Thomas, MD, ${ }^{*} \dagger$ Yeo Jung Park, PhD, ${ }^{*}$ Scott Grey, PhD, ${ }^{*}$ Theodore L Schreiber, MD, $\ddagger$ Hitinder S Gurm, MD, ${ }^{*} \dagger$ Dale Leffler, DO,§ Thomas P Davis, MD,\| Peter Henke, MD, ${ }^{*}+$ P Michael Grossman, $\mathrm{MD}^{*} \dagger$

*University of Michigan, Ann Arbor, MI; †VA Ann Arbor Healthcare System, Ann Arbor, MI; ‡Detroit Medical Center, Detroit, MI; §Sparrow Health System, Lansing, MI; ||Eastlake Cardiovascular, P.C., St Clair Shores, MI

Support: Support for BMC2-VIC is provided by Blue Cross and Blue Shield of Michigan and Blue Care Network as part of the BCBSM Value Partnerships program. The funding source was not involved in any part of this study. Disclaimer: Although

Blue Cross Blue Shield of Michigan and BMC2 work collaboratively, the opinions, beliefs, and viewpoints expressed by the author do not necessarily reflect the opinions, beliefs, and viewpoints of BCBSM or any of its employees.

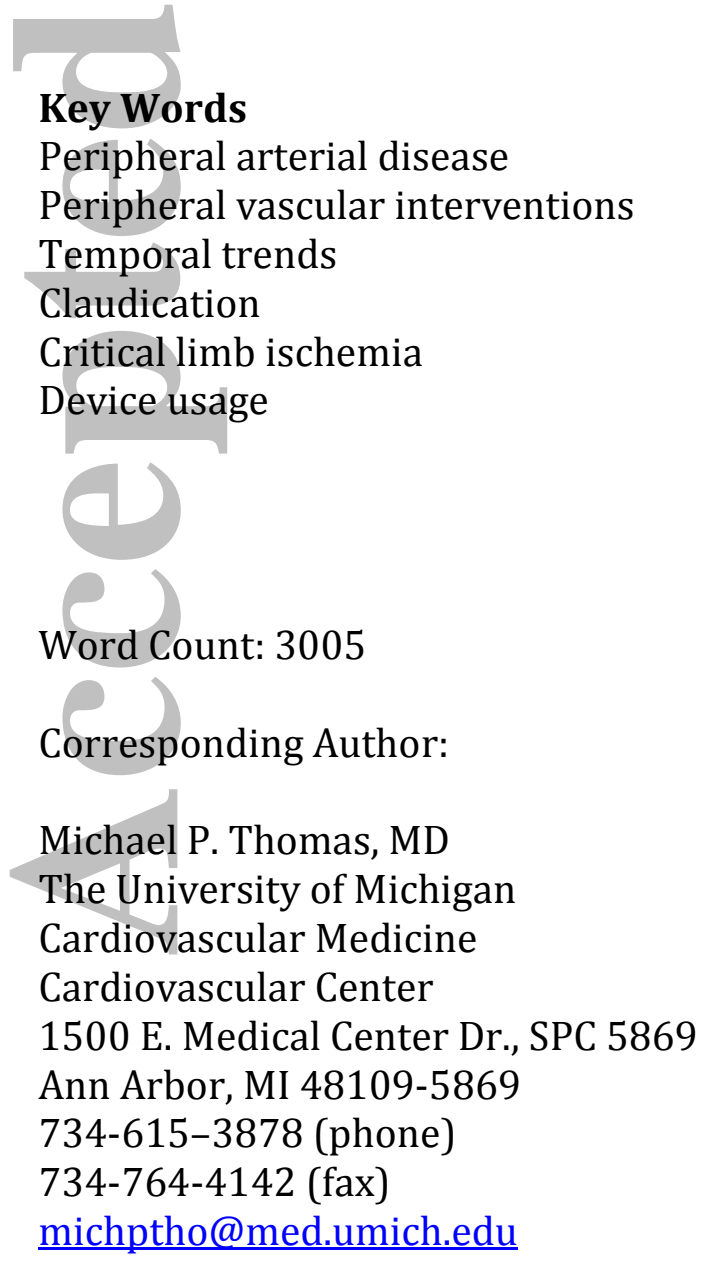

This is the author manuscript accepted for publication and has undergone full peer review but has not been through the copyediting, typesetting, pagination and proofreading process, which may lead to differences between this version and the Version record. Please cite this article as doi:10.1002/ ccd.26883. 


\section{Structured Abstract}

\section{Objectives}

The aim is to examine trends in procedural indication, arterial beds treated, and device usage in peripheral arterial interventions.

\section{Background}

There is little data on indication, vascular beds treated and devices utilized for peripheral arterial interventions (PVIs).

\section{Methods}

We used data from 43 hospitals participating in the BMC2 VIC registry. PVIs were separated by year and divided by arterial segment. Lower extremity PVIs were subclassified as having been performed for claudication or critical limb ischemia.

Yearly device usage was also included. A repeated measure ANOVA was used to determine trends.

\section{Results}

44,650 PVIs were performed from 2006 to 2013 . Renal interventions decreased from $18 \%$ of interventions in 2006 to $5.6 \%$ in $2013(\mathrm{p}<0.001)$ and femoralpopliteal increased from $54.9 \%$ in 2006 to $64.5 \%$ in 2013 ( $p<0.001$ ). No significant trend was seen for aorta-iliac or below-the-knee interventions. $58.6 \%$ of PVIs were performed for claudication in 2006 and this decreased to 44.6\% in 2013 ( $p=0.025$ ). Indications for critical limb ischemia were $24.1 \%$ in 2006 and 47.5\% in 2013 (p < 0.001). There were significant increases in the use of balloon angioplasty ( $\mathrm{p}=$ 0.029) and cutting / scoring balloons ( $<<0.001)$ while cryoballoon usage decreased 
( $p<0.001)$. No significant changes were found with stenting, atherectomy, and laser.

\section{Conclusions}

There is a significant increase in patients presenting with critical limb ischemia.

Renal artery intervention rates are decreasing while femoral-popliteal interventions are increasing. Additionally, balloon angioplasty and cutting / scoring balloon usage (1) is increasing.
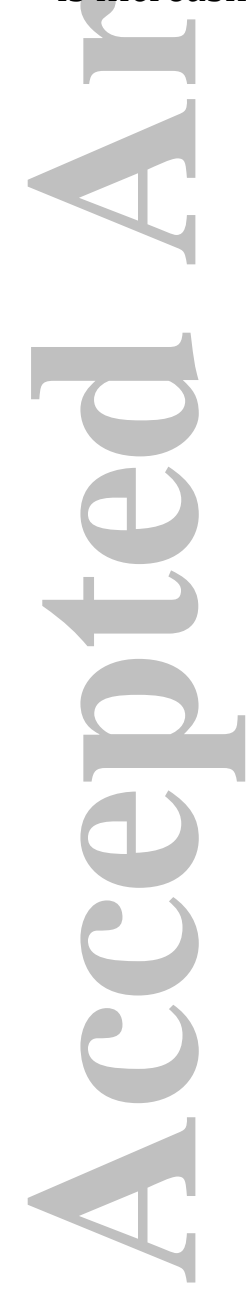


\section{Abbreviations}

$\mathrm{PAD}=$ peripheral arterial disease

$\mathrm{PVI}=$ peripheral vascular interventions

BMC2 PVI = Blue Cross Blue Shield of Michigan Cardiovascular Consortium

Peripheral Vascular Intervention Registry
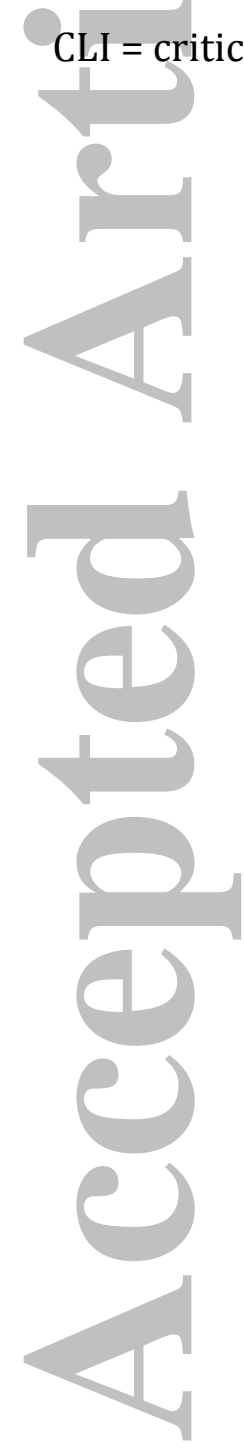


\section{Introduction}

Peripheral arterial disease (PAD) affects approximately 8.5 million Americans $>/=40$ years of age. PAD is associated with significant morbidity and mortality, potential limb loss, and a negative impact on quality of life. The manifestations of PAD are broad and range from asymptomatic to intermittent claudication to critical limb ischemia with tissue loss. (1) In recent years, multisociety efforts have resulted in guidelines for PAD management. (2) Endovascular peripheral vascular interventions (PVI) continue to increase and often offer advantages over surgical intervention. (3) PVIs have become an important part of practice of interventional cardiologists, interventional radiologists and an increasingly utilized approach for vascular surgeons. Additionally, the PVI device market continues to grow. However, there are little contemporary data on the treatment patterns in PVI. It is in this setting that we sought to examine trends in current PVI practice including indication, vascular beds treated, and devices utilized.

\section{Methods}

$\underline{\text { Study Population }}$

The study population consisted of consecutive PVIs (renal, aorto-iliac, femoral-popliteal, and below-the-knee) from 1/2006 to 12/2013 in the Blue Cross Blue Shield of Michigan Cardiovascular Consortium Peripheral Vascular Intervention (BMC2 PVI) Registry. BMC2 PVI Registry 
The details of the BMC2 PVI Registry have been described previously. (4) Briefly, BMC2 PVI is a prospective, multicenter, observational quality improvement registry funded by Blue Cross Blue Shield of Michigan. The registry is a regional collaborative effort aimed to improve the quality of care and patient outcomes and overcome barriers of traditional market and academic competition. The registry collects, audits, and organizes data and reports procedural variables and outcomes to individual operators and institutions. A data form is compiled for each patient, including demographic information, past medical history, laboratories pre- and post-PVI, patient history, presenting symptoms, procedural indications, medication details, PVI types, details of procedure, and associated complications if present. Data quality is ensured by ad hoc queries, random chart reviews, and a series of diagnostic routines included in the database. Peri-procedural and in-hospital data are collected from each individual. The registry has been approved or the need for approval waived by the institutional review board of each participating hospital.

\section{Statistical Analysis}

\section{Location and Indication}

Consecutive PVIs from 1/2006 to 12/2013 were separated by year and were divided by arterial segment (renal, aorto-iliac, femoral-popliteal or below-the-knee) The lower extremity PVIs were sub classified as having been performed secondary to either claudication or critical limb ischemia (CLI) defined as rest pain or ulcer. In order to determine changes in temporal trends, the p-value was calculated using a repeated measure ANOVA.

Device Usage 
PVI devices included balloon, stent, atherectomy, cryoballoon, cutting balloon, and laser. A repeated measure ANOVA was used to determine temporal trends in device usage.

\section{Sensitivity Analysis}

The analysis was repeated utilizing only the original 6 hospitals in the registry from 2006-2013 to ensure that the changes observed were not secondary to a difference in treatment culture within the new centers.

\section{Results}

A total of 44,650 consecutive patients undergoing PVI were prospectively enrolled from 43 hospitals in Michigan state from January 2006 through December 2013 (Table I). Baseline demographic and clinical characteristics are detailed in

Table II. Across any given year, approximately $41-45 \%$ of the patients were female and the cohort had an average age of 68 years-old with a BMI ranging from $28-32$ $\mathrm{kg} / \mathrm{m}^{2}$. Nearly one-third of patients were current smokers and $43.5 \%-58.5 \%$ had a history of smoking. Nearly one-half of patients had a history of diabetes and approximately two-thirds had concomitant coronary artery disease. Additionally, over $80 \%$ of patients had a history of hyperlipidemia while over $90 \%$ had a history of hypertension.

A significant temporal trend was observed for procedural indication from 2006 to 2013 . In $2006,58.6 \%$ of the 1677 PVIs were performed for claudication while $44.6 \%$ of the 9185 PVIs were performed for claudication in 2013 ( $p=0.025)$. 
Critical limb ischemia comprised $24.1 \%$ of the interventions in 2006 and increased to $47.5 \%$ of the PVIs in $2013(\mathrm{p}<0.001)$. (Figure 1)

There were also significant changes in the location of the vascular beds that were treated over the study period. Renal interventions accounted for $18 \%$ of the overall PVIs in 2006 and decreased to $5.6 \%$ of the interventions in $2013(\mathrm{p}<0.001)$.

Femoral-popliteal interventions accounted for 54.9\% of PVIs in 2006 and increased to comprise $64.5 \%$ of the interventions in $2013(\mathrm{p}<0.001)$. No significant trend was seen in the percent of overall cases comprised of aorta-iliac or below-the-knee interventions from 2006 to 2013 (Table III) In 2006, 67.6\% of PVIs utilized balloon angioplasty and this increased to $85.4 \%$ in $2013(p=0.029)$. Cryoballoon usage decreased from $5 \%$ in 2006 to $0.6 \%$ in 2013 ( $p<0.001$ ) while cutting/scoring balloon use increased from $1.8 \%$ in 2006 to $15.1 \%$ in 2013 ( $p<0.001)$. There was no significant temporal trend found in the use of stenting, atherectomy, and laser from 2006 to 2013. (Table IV)

\section{Sensitivity Analysis}

A total of 13,383 consecutive PVIs were performed at the original 6 hospitals in the registry from 2006 through 2013 (Table I). A significant temporal trend was observed for procedural indication as claudication decreased $(\mathrm{p}=0.046)$ and critical limb ischemia increased $(\mathrm{p}<0.001)$. (Supplementary Figure 1) In regards to vascular beds treated, there was a significant decrease in renal artery interventions and increase in femoral-popliteal and below-the-knee interventions while there was no significant trend observed in aorto-iliac interventions. (Supplementary Table I) There were significant increases observed in the use of balloon and cutting 
/scoring balloons while there were significant decreases seen in stenting, cryoballoon and laser therapy. There was no significant trend observed with the use of atherectomy. (Supplementary Table II)

\section{Discussion}

In this large cohort of PVI patients from 2006 to 2013, we observed several changes in indication for the procedure, vascular bed treated and device utilization over the study period. In more recent years, there have been a greater proportion of patients being treated for critical limb ischemia. The rates of renal artery interventions decreased over time, while the rates of femoral-popliteal artery interventions increased. Additionally, balloon angioplasty and cutting balloon usage increased, while cryoballoon therapy declined. Atherosclerotic risk factors were widely prevalent in this cohort and there were no major changes in the patient wasel baseline characteristics.

\section{Procedural Indication}

The key finding of our study is that there has been a significant shift in the type of procedures that are being performed. Over the study period, there was an increase in the PVI procedural indication of critical limb ischemia and decrease in claudication from 2006 through 2013. In 2006, approximately one in four cases (24.1\%) were completed for CLI while this increased to nearly one-half in 2013 $(47.5 \%)$

It is likely that the increased prevalence of CLI as an indication in our study is a reflection of the increasing recognition of the safety and efficacy of percutaneous 
versus surgical revascularization in patients with significant co-morbidities. Nasr et al. reviewed single center institutional revascularization rates for critical limb ischemia from 1994 to 1999 . They found that PTA rates increased from $44 \%$ in 1994-1995 to 69\% in 1998-1999 while surgical rates declined. There was no statistically significant difference between the groups in regards to patient survival, limb salvage rates, and mean hospital stay. (5) Plaisance et al. examined the safety of PVI in the elderly in a statewide registry consisting of 7,769 patients. The authors found that the elderly patients presented with more severe PAD including rest pain or goal of limb salvage, however, after adjustment for baseline covariates, advanced age was not associated with increased rates of MACE, transfusion, contrast induced nephropathy, or amputation. (6) Furthermore, surgical revascularization for critical limb ischemia has been found to be associated with a higher 30-day and 1year mortality in octogenarians when compared to younger patients. (7) Also, Faglia et al. found that percutaneous revascularization was feasible in $84 \%$ of diabetic patients presenting with CLI as a first choice revascularization procedure and had an associated low complication rate (3.4\%). (8) Therefore, it is not surprising that there is increased utilization of PVI for CLI patients..

\section{Arterial Bed Treated}

Renal artery interventions decreased from 2006 to 2013 while rates of

femoral-popliteal increased. There was no significant trend observed in rates of aorto-iliac or below-the-knee interventions during this time period. As shown in Table III, rates of aorto-iliac are relatively stable while the proportion of below-theknee interventions does increase over time, but is not statistically significant. This 
may be secondary to the smaller number of hospitals in the Registry in the earlier time points as one would expect an increase in the below-the-knee interventions with an increased proportion of procedures performed for critical limb ischemia. In our study, the rate of renal artery intervention dropped from 18\% in 2006 to approximately $6 \%$ in 2010 . This percentage remained relatively stable through 2013. Notably, this decrease in the rate of renal artery intervention was prior to the publication of the CORAL study in 2014, which confirmed that effective medical therapy should be the first line of treatment in patients with presumed renovascular hypertension. (9) It is likely that the widespread uncertainty and the clinical equipoise about the efficacy of renal stenting resulted in a decline of this procedure even prior to the publication of the CORAL trial.

Goodney et al. examined trends in lower extremity revascularization practices in Medicare beneficiaries from 1996 to 2006 and found that endovascular treatment increased three-fold during this time period while lower-extremity bypass decreased by $42 \%$. (10) This change in practice may have been partially driven by patient preference for less invasive procedures that paralleled advances in catheter-based technologies. This practice pattern is consistent with our observation of an increase in the rate in femoral-popliteal interventions from 2006 to 2013 .

Device Utilization

Data from our large registry would suggest that plain balloon angioplasty and cutting/scoring balloon angioplasty has increased while cryoballoon therapy is decreased. In fact, the cryoballoon was withdrawn from the U.S. market for a period 
of time and recently reintroduced. Therefore, these rates may begin to increase again. Additionally, there was no significant trend identified in the use of stents, atherectomy devices, or laser from 2006 to 2013. The ACC/AHA guidelines for the management of patients with PAD provide a basic framework for utilization of these devices. (11) Stenting for iliac artery stenosis is a Class I LOE B recommendation while stenting and other adjunct therapies can be useful for salvage therapy from balloon dilatation of femoral, popliteal, or tibial vessels (Class IIb LOE C recommendation).

Along with these basic guidelines, the device market for treatment of PAD continues to expand rapidly and adjunct therapies are more readily available including drug eluting balloons and atherectomy devices. Given the number and variability in treatment modalities, as well as practitioner-preference and the lack of significant clinical trial data, the interventionalist or surgeon has a wide array of devices to chose from in treating in a specific lesion.

To our knowledge, this is the largest study to examine changes in device usage in treatment of peripheral arterial disease.

\section{$\underline{\text { Limitations }}$}

The results in this study are based upon observational data that are not adjudicated by a central facility. The use of a large registry with clinical heterogeneity and reporting variability leads to the possibility for confounding variables and treatment bias. Additionally, all patients in our study underwent PVI at hospitals participating in a quality improvement initiative and therefore, these findings may not apply to other institutions or patients from other areas. However, the data is based on a 
large registry and reflects continuous data on unselected patients undergoing PVI procedures performed by multiple specialists from different backgrounds (interventional cardiology, interventional radiology, vascular surgery) and offers a contemporary insight into treatment in this population.

\section{Conclusions}

In this study, a there was a significant increase in the proportion of patients that presented for lower extremity PVI with critical limb ischemia, while fewer presented with claudication. From 2006-2013, the rates of renal artery interventions decreased while femoral-popliteal interventions increased.

Additionally, the use of balloon angioplasty and cutting/scoring balloon increased from 2006 to 2013. These findings shed light on contemporary practice patterns related to the percutaneous management of PAD and suggest the need for additional stucie studies to define optimal treatment strategies.

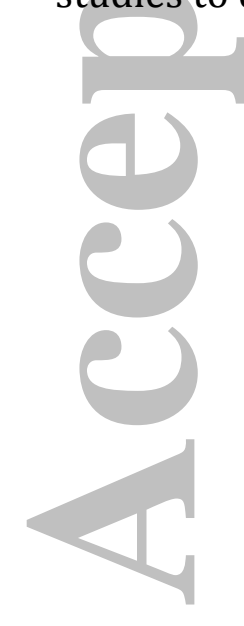




\section{References}

1. Go AS, Mozaffarian D, Roger VL et al. Heart disease and stroke statistics-2014 update: a report from the American Heart Association. Circulation 2014;129:e28-e292.

2. Hirsch AT, Haskal ZJ, Hertzer NR et al. ACC/AHA 2005 guidelines for the management of patients with peripheral arterial disease (lower extremity, renal, mesenteric, and abdominal aortic): executive summary a collaborative report from the American Association for Vascular Surgery/Society for Vascular Surgery, Society for Cardiovascular Angiography and Interventions, Society for Vascular Medicine and Biology, Society of Interventional

Radiology, and the ACC/AHA Task Force on Practice Guidelines (Writing

Committee to Develop Guidelines for the Management of Patients With Peripheral Arterial Disease) endorsed by the American Association of Cardiovascular and Pulmonary Rehabilitation; National Heart, Lung, and Blood Institute; Society for Vascular Nursing; TransAtlantic Inter-Society Consensus; and Vascular Disease Foundation. Journal of the American College of Cardiology 2006;47:1239-312.

3. Nowygrod R, Egorova N, Greco G et al. Trends, complications, and mortality in peripheral vascular surgery. Journal of vascular surgery 2006;43:205-16.

4. Mukherjee D, Munir K, Hirsch AT et al. Development of a multicenter peripheral arterial interventional database: the PVD-QI2. American heart journal 2005;149:1003-8. 
5. Nasr MK, McCarthy RJ, Hardman J, Chalmers A, Horrocks M. The increasing role of percutaneous transluminal angioplasty in the primary management of critical limb ischaemia. European journal of vascular and endovascular surgery : the official journal of the European Society for Vascular Surgery 2002;23:398-403.

6. Plaisance BR, Munir K, Share DA et al. Safety of contemporary percutaneous peripheral arterial interventions in the elderly insights from the BMC2 PVI (Blue Cross Blue Shield of Michigan Cardiovascular Consortium Peripheral Vascular Intervention) registry. JACC Cardiovascular interventions 2011;4:694-701.

7. Brosi P, Dick F, Do DD, Schmidli J, Baumgartner I, Diehm N. Revascularization for chronic critical lower limb ischemia in octogenarians is worthwhile. Journal of vascular surgery 2007;46:1198-207.

8. Faglia E, Dalla Paola L, Clerici G et al. Peripheral angioplasty as the firstchoice revascularization procedure in diabetic patients with critical limb ischemia: prospective study of 993 consecutive patients hospitalized and followed between 1999 and 2003. European journal of vascular and endovascular surgery : the official journal of the European Society for Vascular Surgery 2005;29:620-7.

9. Cooper CJ, Murphy TP, Cutlip DE et al. Stenting and medical therapy for atherosclerotic renal-artery stenosis. The New England journal of medicine 2014;370:13-22. 
10. Goodney PP, Beck AW, Nagle J, Welch HG, Zwolak RM. National trends in lower extremity bypass surgery, endovascular interventions, and major amputations. Journal of vascular surgery 2009;50:54-60.

11. Anderson JL, Halperin JL, Albert NM et al. Management of patients with peripheral artery disease (compilation of 2005 and 2011 ACCF/AHA

guideline recommendations): a report of the American College of Cardiology Foundation/American Heart Association Task Force on Practice Guidelines. Circulation 2013;127:1425-43.

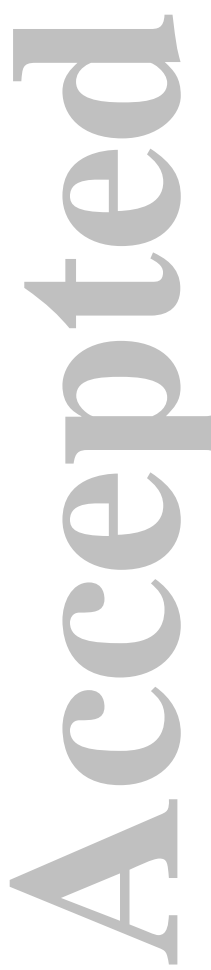




\section{Figure Legend}

Figure 1 - Procedural Indication

Shown is the temporal changes in the proportion of patients undergoing peripheral vascular intervention for claudication and critical limb ischemia.
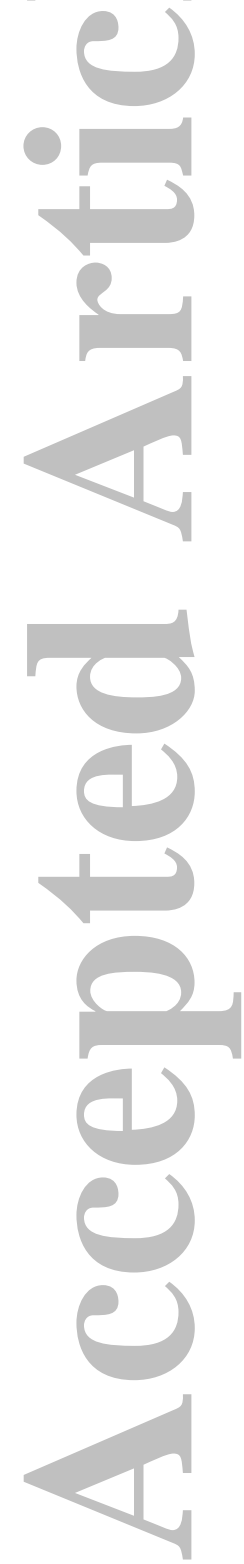
Table I: Hospital Count and Case Number

\begin{tabular}{|l|c|c|c|c|c|c|c|c|c|}
\hline & 2006 & 2007 & 2008 & 2009 & 2010 & 2011 & 2012 & 2013 & Total \\
\hline Discharges & 1677 & 2074 & 3965 & 5395 & 6240 & 7614 & 8500 & 9185 & 44650 \\
\hline $\begin{array}{l}\text { Original 6 } \\
\text { Hospitals* }\end{array}$ & 1677 & 1848 & 1793 & 1636 & 1830 & 1524 & 1323 & 1752 & 13383 \\
\hline Hospital Count & 6 & 7 & 16 & 16 & 18 & 37 & 41 & 43 & \\
\hline
\end{tabular}

${ }^{*}$ Six hospitals that were included since 2006
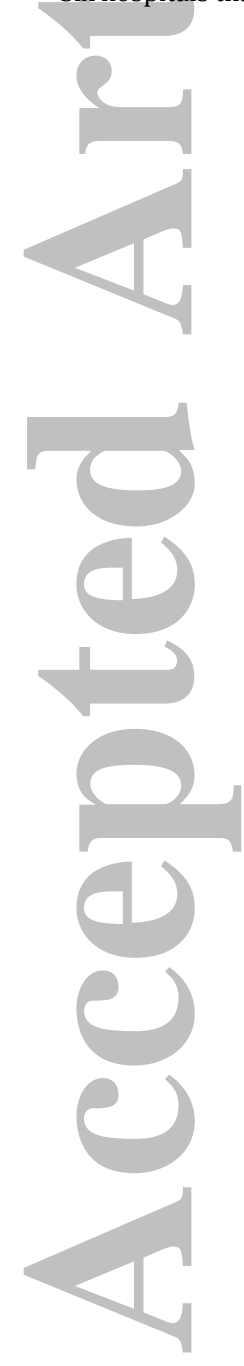
Table II: Baseline Demographic and Clinical Characteristics

\begin{tabular}{|c|c|c|c|c|c|c|c|c|}
\hline & 2006 & 2007 & 2008 & 2009 & 2010 & 2011 & 2012 & 2013 \\
\hline $\mathrm{N}=$ & 1677 & 2074 & 3965 & 5395 & 6240 & 7614 & 8500 & 9185 \\
\hline$\overline{\text { Gend }}$ & $\begin{array}{c}726 \\
(43.4 \%)\end{array}$ & $\begin{array}{c}905 \\
(43.6 \%)\end{array}$ & $\begin{array}{c}1760 \\
(44.4 \%)\end{array}$ & $\begin{array}{c}2403 \\
(44.5 \%)\end{array}$ & $\begin{array}{c}2736 \\
(43.9 \%)\end{array}$ & $\begin{array}{c}3319 \\
(43.6 \%)\end{array}$ & $\begin{array}{c}3622 \\
(42.6 \%)\end{array}$ & $\begin{array}{c}3786 \\
(41.2 \%)\end{array}$ \\
\hline Never Sm & $\begin{array}{l}1324 \\
(79 \%)\end{array}$ & $\begin{array}{c}1669 \\
(80.5 \%)\end{array}$ & $\begin{array}{c}3117 \\
(78.6 \%)\end{array}$ & $\begin{array}{c}4055 \\
(75.2 \%)\end{array}$ & $\begin{array}{c}4840 \\
(77.6 \%)\end{array}$ & $\begin{array}{c}6025 \\
(79.1 \%)\end{array}$ & $\begin{array}{c}6999 \\
(82.3 \%)\end{array}$ & $\begin{array}{c}7703 \\
(83.9 \%)\end{array}$ \\
\hline $\begin{array}{l}\text { Current } \\
\text { Smoker }\end{array}$ & $\begin{array}{c}455 \\
(27.1 \%)\end{array}$ & $\begin{array}{c}626 \\
(30.2 \%)\end{array}$ & $\begin{array}{c}1166 \\
(29.4 \%)\end{array}$ & $\begin{array}{c}1736 \\
(32.2 \%)\end{array}$ & $\begin{array}{c}2096 \\
(33.6 \%)\end{array}$ & $\begin{array}{c}2499 \\
(32.8 \%)\end{array}$ & $\begin{array}{c}2846 \\
(33.5 \%)\end{array}$ & $\begin{array}{c}3223 \\
(35.1 \%)\end{array}$ \\
\hline $\begin{array}{l}\text { Former } \\
\text { Smoker }\end{array}$ & $\begin{array}{c}981 \\
(58.5 \%)\end{array}$ & $\begin{array}{c}1128 \\
(54.4 \%)\end{array}$ & $\begin{array}{c}1961 \\
(49.5 \%)\end{array}$ & $\begin{array}{c}2347 \\
(43.5 \%)\end{array}$ & $\begin{array}{c}2782 \\
(44.6 \%)\end{array}$ & $\begin{array}{l}3563 \\
(46.8)\end{array}$ & $\begin{array}{l}4167 \\
(49 \%)\end{array}$ & $\begin{array}{c}4596 \\
(50 \%)\end{array}$ \\
\hline Age (mean) & 69.2 & 68.1 & 68.8 & 68.5 & 68.3 & 68.6 & 68.4 & 68.4 \\
\hline BMI (mean) & 30.4 & 31.5 & 30.1 & 28.5 & 28.3 & 28.2 & 28.4 & 28.9 \\
\hline $\begin{array}{l}\text { Family } \mathrm{Hx} \text { of } \\
\text { CAD }\end{array}$ & $\begin{array}{c}500 \\
(29.8 \%)\end{array}$ & $\begin{array}{c}620 \\
(29.9 \%)\end{array}$ & $\begin{array}{c}1043 \\
(26.3 \%)\end{array}$ & $\begin{array}{c}1439 \\
(26.7 \%)\end{array}$ & $\begin{array}{c}1610 \\
(25.8 \%)\end{array}$ & $\begin{array}{c}1955 \\
(25.7 \%)\end{array}$ & $\begin{array}{l}1617 \\
(19 \%)\end{array}$ & $\begin{array}{c}1841 \\
(20 \%)\end{array}$ \\
\hline Hyperlipidemia & $\begin{array}{c}1372 \\
(81.8 \%)\end{array}$ & $\begin{array}{c}1753 \\
(84.5 \%)\end{array}$ & $\begin{array}{c}3394 \\
(85.6 \%)\end{array}$ & $\begin{array}{c}4494 \\
(83.3 \%)\end{array}$ & $\begin{array}{c}5442 \\
(87.2 \%)\end{array}$ & $\begin{array}{c}6598 \\
(86.7 \%)\end{array}$ & $\begin{array}{l}7391 \\
(87 \%)\end{array}$ & $\begin{array}{c}8075 \\
(87.9 \%)\end{array}$ \\
\hline Hyper & $\begin{array}{c}1525 \\
(90.9 \%)\end{array}$ & $\begin{array}{c}1920 \\
(92.6 \%)\end{array}$ & $\begin{array}{c}3626 \\
(91.5 \%)\end{array}$ & $\begin{array}{c}4944 \\
(91.6 \%)\end{array}$ & $\begin{array}{c}5738 \\
(92 \%)\end{array}$ & $\begin{array}{c}6968 \\
(91.5 \%)\end{array}$ & $\begin{array}{c}7776 \\
(91.5 \%)\end{array}$ & $\begin{array}{c}8419 \\
(91.7 \%)\end{array}$ \\
\hline Diab & $\begin{array}{c}720 \\
(42.9 \%)\end{array}$ & $\begin{array}{c}953 \\
(46 \%)\end{array}$ & $\begin{array}{c}1851 \\
(46.7 \%)\end{array}$ & $\begin{array}{c}2552 \\
(47.3 \%)\end{array}$ & $\begin{array}{c}2914 \\
(46.7 \%)\end{array}$ & $\begin{array}{c}3525 \\
(46.3 \%)\end{array}$ & $\begin{array}{c}4119 \\
(48.5 \%)\end{array}$ & $\begin{array}{c}4517 \\
(49.2 \%)\end{array}$ \\
\hline Histor & $\begin{array}{c}352 \\
(21 \%)\end{array}$ & $\begin{array}{c}473 \\
(22.8 \%)\end{array}$ & $\begin{array}{c}787 \\
(19.9 \%)\end{array}$ & $\begin{array}{l}1080 \\
(20 \%)\end{array}$ & $\begin{array}{c}1238 \\
(19.8 \%)\end{array}$ & $\begin{array}{l}1521 \\
(20 \%)\end{array}$ & $\begin{array}{c}1793 \\
(21.1 \%)\end{array}$ & $\begin{array}{c}2099 \\
(22.9 \%)\end{array}$ \\
\hline $\begin{array}{l}\text { Significant } \\
\text { valve disease }\end{array}$ & $76(4.5 \%)$ & $\begin{array}{c}171 \\
(8.2 \%)\end{array}$ & $238(6 \%)$ & $\begin{array}{c}347 \\
(6.4 \%)\end{array}$ & $\begin{array}{c}447 \\
(7.2 \%)\end{array}$ & $\begin{array}{c}323 \\
(4.2 \%)\end{array}$ & $680(8 \%)$ & $\begin{array}{c}729 \\
(7.9 \%)\end{array}$ \\
\hline COPD & $\begin{array}{c}446 \\
(26.6 \%)\end{array}$ & $\begin{array}{c}553 \\
(26.7 \%)\end{array}$ & $\begin{array}{c}1130 \\
(28.5 \%)\end{array}$ & $\begin{array}{c}1519 \\
(28.2 \%)\end{array}$ & $\begin{array}{c}1764 \\
(28.3 \%)\end{array}$ & $\begin{array}{c}1912 \\
(25.1 \%)\end{array}$ & $\begin{array}{c}2423 \\
(28.5 \%)\end{array}$ & $\begin{array}{c}2742 \\
(29.9 \%)\end{array}$ \\
\hline CVD or TIA & $\begin{array}{c}484 \\
(28.9 \%)\end{array}$ & $\begin{array}{c}670 \\
(32.3 \%)\end{array}$ & $\begin{array}{c}1208 \\
(30.5 \%)\end{array}$ & $\begin{array}{c}1583 \\
(29.3 \%)\end{array}$ & $\begin{array}{c}1864 \\
(29.9 \%)\end{array}$ & $\begin{array}{c}2150 \\
(28.2 \%)\end{array}$ & $\begin{array}{c}2434 \\
(28.6 \%)\end{array}$ & $\begin{array}{c}2696 \\
(29.4 \%)\end{array}$ \\
\hline Hx of CAD & $\begin{array}{c}1131 \\
(67.4 \%)\end{array}$ & $\begin{array}{c}1394 \\
(67.2 \%)\end{array}$ & $\begin{array}{c}2491 \\
(62.8 \%)\end{array}$ & $\begin{array}{l}3399 \\
(63 \%)\end{array}$ & $\begin{array}{c}3921 \\
(62.8 \%)\end{array}$ & $\begin{array}{c}4554 \\
(59.8 \%)\end{array}$ & $\begin{array}{c}5094 \\
(59.9 \%)\end{array}$ & $\begin{array}{c}5473 \\
(59.6 \%)\end{array}$ \\
\hline Previous PCI & 587 & 763 & 1279 & 1671 & 2076 & 2323 & 2743 & 2956 \\
\hline
\end{tabular}

\section{Catheterization and Cardiovascular Interventions}

This article is protected by copyright. All rights reserved. 


\begin{tabular}{|l|c|c|c|c|c|c|c|c|}
\hline & $(35 \%)$ & $(36.8 \%)$ & $(32.3 \%)$ & $(31 \%)$ & $(33.3 \%)$ & $(30.5 \%)$ & $(32.3 \%)$ & $(32.2 \%)$ \\
\hline Previous MI & 585 & 793 & 1377 & 1527 & 1770 & 2123 & 2412 & 2673 \\
& $(34.9 \%)$ & $(38.2 \%)$ & $(34.7 \%)$ & $(28.3 \%)$ & $(28.4 \%)$ & $(27.9 \%)$ & $(28.4 \%)$ & $(29.1 \%)$ \\
\hline Previous CABG & 474 & 638 & 1180 & 1421 & 1575 & 1859 & 2067 & 2176 \\
& $(28.3 \%)$ & $(30.8 \%)$ & $(29.8 \%)$ & $(26.3 \%)$ & $(25.2 \%)$ & $(24.4 \%)$ & $(24.3 \%)$ & $(23.7 \%)$ \\
\hline GI Bleed & 24 & 40 & 61 & 80 & $97(1.6 \%)$ & $70(0.9 \%)$ & 154 & 189 \\
\hline Atrial & $(1.43 \%)$ & $(1.93 \%)$ & $(1.54 \%)$ & $(1.48 \%)$ & & & $(1.8 \%)$ & $(2.1 \%)$ \\
\hline Fibrillation & $(14 \%)$ & $(14.1 \%)$ & $(13.4 \%)$ & $(13.3 \%)$ & $(12.9 \%)$ & $(13.1 \%)$ & $(14.1 \%)$ & $(15.1 \%)$ \\
\hline Other AVD & $1(0.1 \%)$ & 1046 & 1395 & 1309 & 931 & 1398 & 1950 & 2493 \\
\hline Renal Failure & $78(4.7 \%)$ & 102 & 187 & 253 & 363 & 398 & 491 & $(22.9 \%)$ \\
\hline CRD & & $(4.9 \%)$ & $(4.7 \%)$ & $(4.7 \%)$ & $(5.8 \%)$ & $(5.2 \%)$ & $(5.8 \%)$ & $(5.8 \%)$ \\
\hline Renal & $0(0 \%)$ & $9(0.4 \%)$ & $27(0.7 \%)$ & $53(1 \%)$ & $59(1 \%)$ & $80(1.1 \%)$ & $76(0.9 \%)$ & $96(1.1 \%)$ \\
\hline
\end{tabular}

Abbreviations: $\mathrm{F}$ = female; $\mathrm{BMI}=$ body mass index; $\mathrm{Hx}=$ history; $\mathrm{CAD}=$ coronary

artery disease; $\mathrm{CHF}=$ congestive heart failure; $\mathrm{COPD}=$ chronic obstructive

pulmonary disease; CVD = cerebrovascular disease; TIA = transient ischemic attack;

$\mathrm{PCI}=$ percutaneous coronary intervention; $\mathrm{MI}=$ myocardial infarction; $\mathrm{CABG}=$ coronary artery bypass grafting; GI = gastrointestinal; $\mathrm{AVD}=$; CRD = chronic renal disease 
Table III: Arterial Bed Treated

\begin{tabular}{|l|c|c|c|c|c|c|c|c|c|}
\hline & 2006 & 2007 & 2008 & 2009 & 2010 & 2011 & 2012 & 2013 & $\begin{array}{c}\text { P-value } \\
\text { for trend }\end{array}$ \\
\hline Renal & 301 & 260 & 416 & 439 & 360 & 460 & 482 & 514 & $<.001$ \\
& $(18 \%)$ & $(12.5 \%)$ & $(10.5 \%)$ & $(8.1 \%)$ & $(5.8 \%)$ & $(6 \%)$ & $(5.7 \%)$ & $(5.6 \%)$ & \\
\hline Aorto- & 482 & 597 & 1165 & 1485 & 1737 & 2124 & 2340 & 2555 & 0.295 \\
Iliac & $(28.7 \%)$ & $(28.8 \%)$ & $(29.4 \%)$ & $(27.5 \%)$ & $(27.8 \%)$ & $(27.9 \%)$ & $(27.5 \%)$ & $(27.8 \%)$ & \\
\hline Femoral- & 921 & 1238 & 2380 & 3324 & 3910 & 4732 & 5593 & 5926 & $<.001$ \\
Popliteal & $(54.9 \%)$ & $(59.7 \%)$ & $(60 \%)$ & $(61.6 \%)$ & $(62.7 \%)$ & $(62.2 \%)$ & $(65.8 \%)$ & $(64.5 \%)$ & \\
\hline Below & 261 & 429 & 968 & 1466 & 1652 & 2061 & 2181 & 2396 & 0.092 \\
Knee & $(15.6 \%)$ & $(20.7 \%)$ & $(24.4 \%)$ & $(27.2 \%)$ & $(26.5 \%)$ & $(27.1 \%)$ & $(25.7 \%)$ & $(26.1 \%)$ & \\
\hline
\end{tabular}

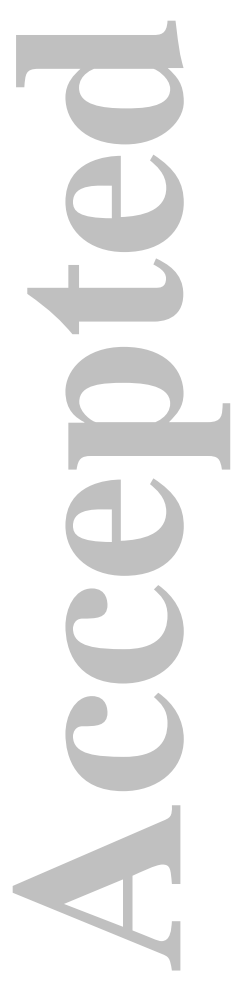


Table IV: Device Usage

\begin{tabular}{|c|c|c|c|c|c|c|c|c|c|}
\hline & 2006 & 2007 & 2008 & 2009 & 2010 & 2011 & 2012 & 2013 & $\begin{array}{c}\text { P-value } \\
\text { for } \\
\text { trend }\end{array}$ \\
\hline Bal & $\begin{array}{c}1134 \\
(67.6 \%)\end{array}$ & $\begin{array}{c}1537 \\
(74.1 \%)\end{array}$ & $\begin{array}{c}3263 \\
(82.3 \%)\end{array}$ & $\begin{array}{c}4670 \\
(86.6 \%)\end{array}$ & $\begin{array}{c}5529 \\
(88.6 \%)\end{array}$ & $\begin{array}{c}6745 \\
(88.6 \%)\end{array}$ & $\begin{array}{c}7434 \\
(87.5 \%)\end{array}$ & $\begin{array}{c}7845 \\
(85.4 \%)\end{array}$ & 0.029 \\
\hline Stent & $\begin{array}{c}932 \\
(55.6 \%)\end{array}$ & $\begin{array}{c}1106 \\
(53.3 \%)\end{array}$ & $\begin{array}{c}2059 \\
(51.9 \%)\end{array}$ & $\begin{array}{c}2477 \\
(45.9 \%)\end{array}$ & $\begin{array}{c}2752 \\
(44.1 \%)\end{array}$ & $\begin{array}{c}3530 \\
(46.4 \%)\end{array}$ & $\begin{array}{c}3969 \\
(46.7 \%)\end{array}$ & $\begin{array}{c}4146 \\
(45.1 \%)\end{array}$ & 0.131 \\
\hline Atherectomy & $\begin{array}{c}401 \\
(23.9 \%)\end{array}$ & $\begin{array}{c}559 \\
(27 \%)\end{array}$ & $\begin{array}{c}1218 \\
(30.7 \%)\end{array}$ & $\begin{array}{c}1743 \\
(32.3 \%)\end{array}$ & $\begin{array}{c}1961 \\
(31.4 \%)\end{array}$ & $\begin{array}{c}2113 \\
(27.8 \%)\end{array}$ & $\begin{array}{c}2114 \\
(24.9 \%)\end{array}$ & $\begin{array}{c}2127 \\
(23.2 \%)\end{array}$ & 0.558 \\
\hline Cryoballoon & $83(5 \%)$ & $\begin{array}{c}74 \\
(3.6 \%)\end{array}$ & $\begin{array}{c}122 \\
(3.1 \%)\end{array}$ & $\begin{array}{l}215 \\
(4 \%)\end{array}$ & $\begin{array}{c}213 \\
(3.4 \%)\end{array}$ & $\begin{array}{c}155 \\
(2 \%)\end{array}$ & $\begin{array}{c}136 \\
(1.6 \%)\end{array}$ & $\begin{array}{c}59 \\
(0.6 \%)\end{array}$ & $<.001$ \\
\hline $\begin{array}{l}\text { Cutting } \\
\text { Balloon }\end{array}$ & $\begin{array}{c}30 \\
(1.8 \%)\end{array}$ & $\begin{array}{c}43 \\
(2.1 \%)\end{array}$ & $\begin{array}{c}146 \\
(3.7 \%)\end{array}$ & $\begin{array}{c}174 \\
(3.2 \%)\end{array}$ & $\begin{array}{c}302 \\
(4.8 \%)\end{array}$ & $\begin{array}{c}550 \\
(7.2 \%)\end{array}$ & $\begin{array}{c}1068 \\
(12.6 \%)\end{array}$ & $\begin{array}{c}1388 \\
(15.1 \%)\end{array}$ & $<.001$ \\
\hline Laser & $\begin{array}{c}87 \\
(5.2 \%)\end{array}$ & $\begin{array}{c}189 \\
(9.1 \%)\end{array}$ & $\begin{array}{c}436 \\
(11 \%)\end{array}$ & $\begin{array}{c}631 \\
(11.7 \%)\end{array}$ & $\begin{array}{c}584 \\
(9.4 \%)\end{array}$ & $\begin{array}{c}555 \\
(7.3 \%)\end{array}$ & $\begin{array}{c}484 \\
(5.7 \%)\end{array}$ & $\begin{array}{c}676 \\
(7.4 \%)\end{array}$ & 0.298 \\
\hline
\end{tabular}

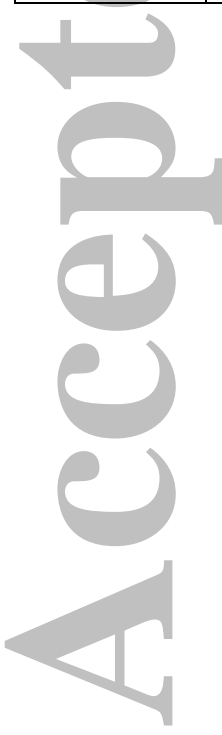


Figure 1: Procedural Indication

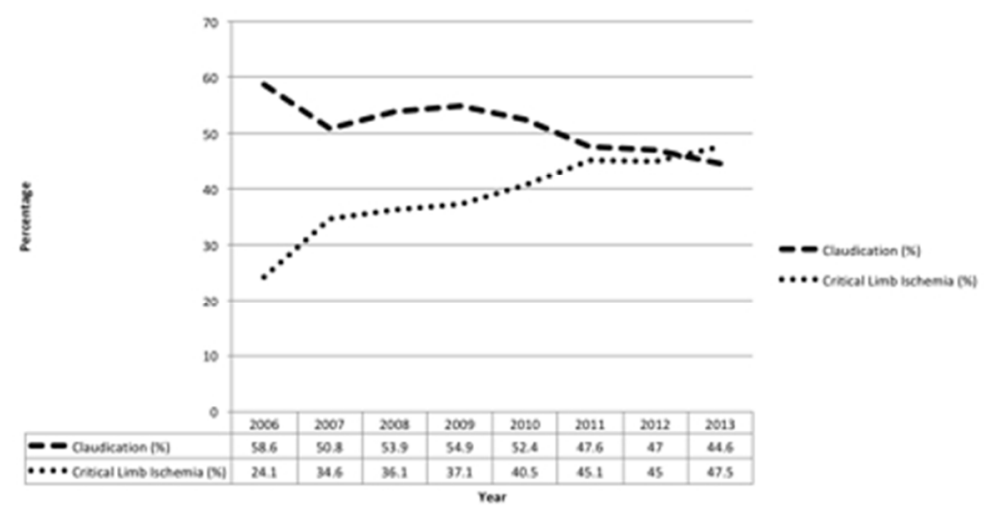

Caption: Shown is the temporal changes in the proportion of patients undergoing peripheral vascular intervention for claudication and critical limb ischemia.

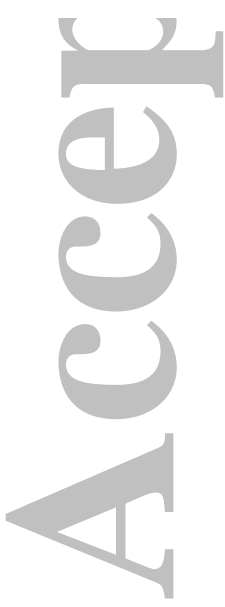

$200 \times 157 \mathrm{~mm}(96 \times 96 \mathrm{DPI})$ 
Supplementary Table I: Arterial Bed Treated in Original 6 Hospitals

\begin{tabular}{|l|c|c|c|c|c|c|c|c|c|}
\hline & 2006 & 2007 & 2008 & 2009 & 2010 & 2011 & 2012 & 2013 & $\begin{array}{c}\text { P-value } \\
\text { for trend }\end{array}$ \\
\hline Renal & 301 & 230 & 211 & 174 & 105 & 112 & 72 & 88 & $<.001$ \\
& $(18 \%)$ & $(12.5 \%)$ & $(11.8 \%)$ & $(10.6 \%)$ & $(5.7 \%)$ & $(7.4 \%)$ & $(5.4 \%)$ & $(5.0 \%)$ & \\
\hline Aorto- & 482 & 527 & 578 & 534 & 596 & 501 & 435 & 532 & 0.451 \\
Iliac & $(28.7 \%)$ & $(28.5 \%)$ & $(32.2 \%)$ & $(32.6 \%)$ & $(32.6 \%)$ & $(32.9 \%)$ & $(32.9 \%)$ & $(30.4 \%)$ & \\
\hline Femoral- & 921 & 1117 & 1015 & 922 & 1109 & 883 & 829 & 1132 & $<.001$ \\
\hline Popliteal & $(54.9 \%)$ & $(60.4 \%)$ & $(56.6 \%)$ & $(56.4 \%)$ & $(60.6 \%)$ & $(57.9 \%)$ & $(62.7 \%)$ & $(64.6 \%)$ & \\
\hline Below & 261 & 373 & 382 & 327 & 349 & 292 & 253 & 320 & 0.023 \\
Knee & $(15.6 \%)$ & $(20.2 \%)$ & $(21.3 \%)$ & $(20.0 \%)$ & $(19.1 \%)$ & $(19.2 \%)$ & $(19.1 \%)$ & $(18.3 \%)$ & \\
\hline
\end{tabular}
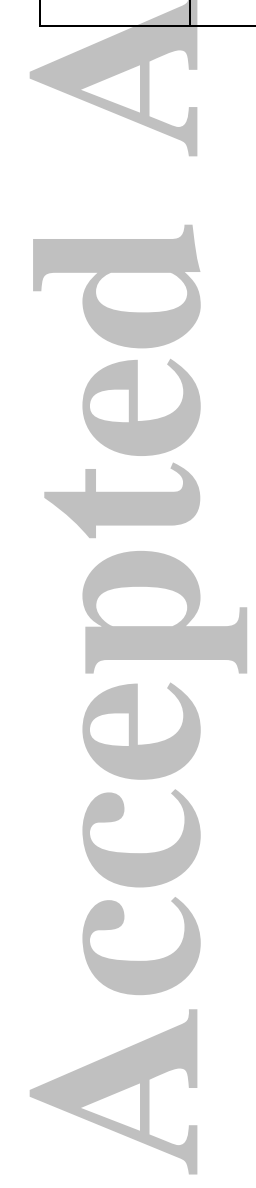
Supplementary Table II: Device Usage in Original 6 Hospitals

\begin{tabular}{|c|c|c|c|c|c|c|c|c|c|}
\hline & 2006 & 2007 & 2008 & 2009 & 2010 & 2011 & 2012 & 2013 & $\begin{array}{l}\text { P-value } \\
\text { for } \\
\text { trend }\end{array}$ \\
\hline Balloon & $\begin{array}{c}1134 \\
(67.6 \%)\end{array}$ & $\begin{array}{c}1338 \\
(72.4 \%)\end{array}$ & $\begin{array}{c}1476 \\
(82.3 \%)\end{array}$ & $\begin{array}{c}1386 \\
(84.7 \%)\end{array}$ & $\begin{array}{c}1609 \\
(87.9 \%)\end{array}$ & $\begin{array}{c}1340 \\
(87.7 \%)\end{array}$ & $\begin{array}{c}1129 \\
(85.3 \%)\end{array}$ & $\begin{array}{c}1473 \\
(84.1 \%)\end{array}$ & 0.011 \\
\hline Stent & $\begin{array}{c}932 \\
(55.6 \%)\end{array}$ & $\begin{array}{c}979 \\
(53.0 \%)\end{array}$ & $\begin{array}{c}997 \\
(55.6 \%)\end{array}$ & $\begin{array}{c}880 \\
(53.8 \%)\end{array}$ & $\begin{array}{c}898 \\
(49.1 \%)\end{array}$ & $\begin{array}{c}807 \\
(53.0 \%)\end{array}$ & $\begin{array}{c}672 \\
(50.8 \%)\end{array}$ & $\begin{array}{c}831 \\
(47.4 \%)\end{array}$ & 0.04 \\
\hline Atherectomy & $\begin{array}{c}401 \\
(23.9 \%)\end{array}$ & $\begin{array}{c}518 \\
(29 \%)\end{array}$ & $\begin{array}{c}458 \\
(25.5 \%)\end{array}$ & $\begin{array}{c}308 \\
(18.8 \%)\end{array}$ & $\begin{array}{c}388 \\
(21.2 \%)\end{array}$ & $\begin{array}{c}201 \\
(13.2 \%)\end{array}$ & $\begin{array}{c}208 \\
(15.7 \%)\end{array}$ & $\begin{array}{c}275 \\
(15.7 \%)\end{array}$ & 0.769 \\
\hline Cryoballoon & $\begin{array}{c}83 \\
(5 \%)\end{array}$ & $\begin{array}{c}74 \\
(4 \%)\end{array}$ & $\begin{array}{c}72 \\
(4 \%)\end{array}$ & $\begin{array}{c}29 \\
(1.8 \%)\end{array}$ & $\begin{array}{c}64 \\
(3.5 \%)\end{array}$ & $\begin{array}{c}36 \\
(2.4 \%)\end{array}$ & $\begin{array}{c}19 \\
(1.4 \%)\end{array}$ & $\begin{array}{c}13 \\
(0.74 \%)\end{array}$ & 0.016 \\
\hline $\begin{array}{l}\text { Cutting } \\
\text { Balloon }\end{array}$ & $\begin{array}{c}30 \\
(1.8 \%)\end{array}$ & $\begin{array}{c}31 \\
(1.7 \%)\end{array}$ & $\begin{array}{c}64 \\
(3.6 \%)\end{array}$ & $\begin{array}{c}51 \\
(3.1 \%)\end{array}$ & $\begin{array}{c}133 \\
(7.3 \%)\end{array}$ & $\begin{array}{c}94 \\
(6.2 \%)\end{array}$ & $\begin{array}{c}114 \\
(8.6 \%)\end{array}$ & $\begin{array}{c}194 \\
(11.1 \%)\end{array}$ & $<.001$ \\
\hline Laser & $\begin{array}{c}87 \\
(5.2 \%)\end{array}$ & $\begin{array}{c}114 \\
(6.2 \%)\end{array}$ & $\begin{array}{c}52 \\
(3 \%)\end{array}$ & $\begin{array}{c}29 \\
(1.8 \%)\end{array}$ & $\begin{array}{c}42 \\
(2.3 \%)\end{array}$ & $\begin{array}{c}19 \\
(1.3 \%)\end{array}$ & $\begin{array}{c}10 \\
(0.8 \%)\end{array}$ & $\begin{array}{c}17 \\
(1 \%)\end{array}$ & 0.038 \\
\hline
\end{tabular}


Supplementary Figure 1: Procedural Indication for Original 6 Hospitals

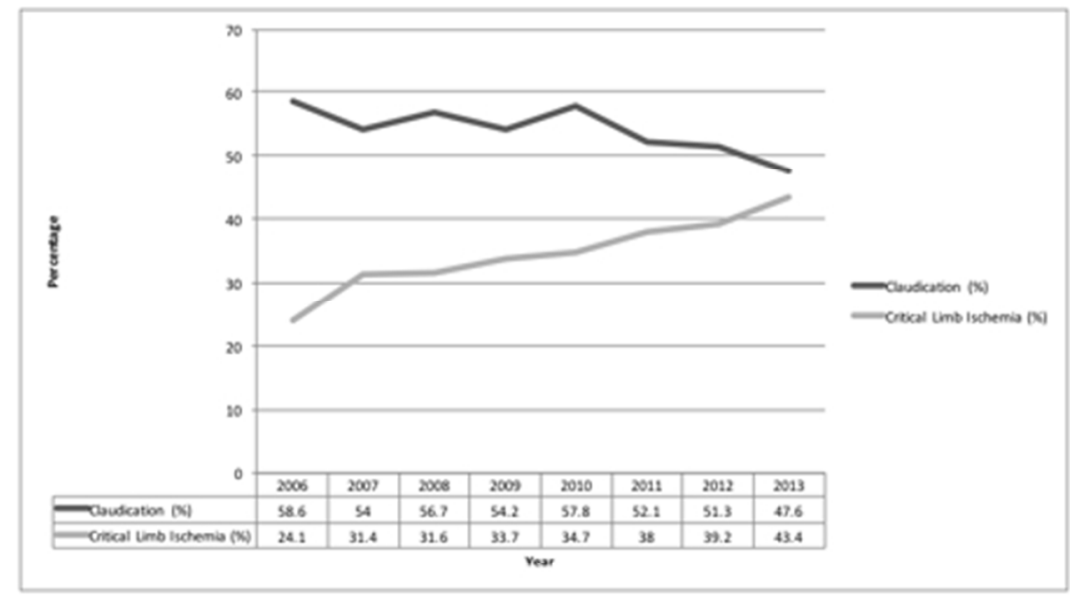

Caption: Shown is the temporal changes in the proportion of patients undergoing peripheral vascular intervention for claudication and critical limb ischemia for the original 6 hospitals.

$192 \times 174 \mathrm{~mm}(96 \times 96 \mathrm{DPI})$

Catheterization and Cardiovascular Interventions

This article is protected by copyright. All rights reserved. 
Please note that this manuscript was submitted twice to JACC Interventions. The first set of comments are from the original submission and second set at the end of this document are from the revised submission. Additionally, our responses to there questions are in our letter to the editor.

Thank you,

Michael P. Thomas

Temporal Trends in Peripheral Arterial Interventions: Observations from the Blue Cross Blue Shield of Michigan Cardiovascular Consortium (BMC2 PVI

Dear Dr. Thomas,

Thank you for your recent submission to JACC: Cardiovascular Interventions. Your manuscript has been carefully evaluated by the editors and by expert external referees. Unfortunately the consensus is that it cannot be accepted. However, we would be willing to consider a revised manuscript on a de novo basis. Because of the extensive revisions required, your manuscript will be treated as a new submission, assigned a new manuscript number, and will be subjected to peer review and prioritization in competition with all other manuscripts.

If you elect to resubmit, please provide a detailed list of all changes made to the manuscript, keyed to the reviewers' comments. In resubmitting please go to www.jaccsubmit-interventions.org. You may contact us by email at jaccint@acc.org if you require assistance. Please state in your cover letter that this is a de novo submission, AND INCLUDE THE ORIGINAL MANUSCRIPT NUMBER.

Thank you for your interest in the journal, and we look forward to reviewing other submissions from you in the future.

Sincerely,

Spencer B. King III, MD, MACC

Editor-in-Chief

JACC: Cardiovascular Interventions

Heart House, 2400 N Street NW, Washington, DC, 20037

$\mathrm{Ph}: 202-375-6136$

Fax: 202-375-6819

Email:jaccint@acc.org

Reviewer \#1 (Comments for the Author (Required)):

In the current manuscript, the authors investigate temporal trends in peripheral vascular interventions and device usage from 2006-2013 based on 43 hospitals participating in 
the Michigan BMC2 VIC registry. The study identified several trends, including decreased rates of renal artery stenting, increased treatment of femoropopliteal disease, and a greater proportion of patients with critical limb ischemia being treated. Device usage was also investigated, with trends for increased balloon angioplasty and less cryoplasty, but reportedly no change in stent rates, atherectomy, or laser. These temporal trends are important and add value to the existing data on procedural trends, but I have several questions related to the methodology and results:

1. In the introduction, the statement that PVI offers a mortality benefit over surgery is overstated. While it is true that PVI is lower risk from an intervention standpoint acutely, no long-term outcomes have demonstrated a mortality benefit (and in fact the BASIL study suggested a benefit of surgery among patients who lived two years).

2. Why were PVIs defined as renal and leg interventions? This seems rather arbitrary, because if renal interventions are included then carotid, subclavian and mesenteric interventions should probably be included. Otherwise the manuscript would be more focused if just focused on aorto-iliac, femoropopliteal, and below the knee disease.

3. What is the documentation or criteria provided for claudication, rest pain, or arterial ulcer in the database? For example, in the absence of hemodynamic criteria, rest pain can be a subjective indication for intervention.

4. The Results showing increased CLI interventions over time but no increase in below knee interventions seem discordant. If there were more CLI interventions, there should have also been more below knee interventions.

5. It is hard to determine how a peripheral intervention would be performed without balloon angioplasty. How is this defined? As stand-alone balloon angioplasty?

6. Laser is a type of atherectomy, so it seems odd that laser is separated out in the abstract, Methods and Results.

7. Many peripheral vascular procedures necessitate the use of combined devices, e.g., atherectomy, balloon angioplasty, and then stenting. How was this accounted for in the analysis? It would be helpful as well to have more Results regarding combination of these therapies, especially in the femoropopliteal segment.

8. Cryoballoons are available again on the market, so the discussion should be revised to reflect that fact.

Reviewer \#2 (Comments for the Author (Required)):

Thomas et al. report the temporal trends in peripheral interventions (renal and leg) from 
a large Michigan registry during years 2006-2013. They identified that renal interventions declined significantly, while femoropopliteal procedures increased. Procedures for critical limb ischemia nearly doubled during this time period.

Please address the following comments/questions:

1) Does the BCBS registry include all PVI procedures or just those of patients with this private insurance?

2) How were multilevel procedures (eg simultaneous fempop plus below knee intervention for a patient with CLI) handled for the purposes of analysis? It is common for below-knee interventions to be performed concurrently with more proximal interventions.

3) Do you have any data on interventions done for restenosis? I wonder if some of the trends, particularly those related to the femoropopliteal segment, reflect treatment of restenosis and not necessarily new patients.

4) It is somewhat discordant to note that the CLI indication nearly doubled, but a significant increase in below-knee interventions was not detected. A large proportion of $\mathrm{CLI}$ patients get below-knee revascularization. Is this simply a power/sample size limitation? Is there any concern about the accuracy of the reporting within the registrywhere leg interventions are being given $C L I$ indications when really just claudication was present (eg operator gaming)?

\footnotetext{
"Temporal Trends in Peripheral Arterial Interventions: Observations from the Blue Cross Blue Shield of Michigan Cardiovascular Consortium (BMC2 PVI)"
}

Dear Dr. Thomas,

Thank you for your recent manuscript submission to JACC: Cardiovascular Interventions. Unfortunately, after careful consideration by the editors and by expert external reviewers, the consensus is that its priority is not sufficient to warrant publication.

The comments of the reviewers are enclosed for your information.

We recognize the thought and effort that went into your work. Regrettably, we are able to publish less than one-fifth of the papers we receive, and must decline many of considerable merit. Thank you for your interest in the journal, and we look forward to reviewing other submissions from you in the future.

Sincerely,

Spencer B. King III, MD, MACC

Editor-in-Chief

JACC: Cardiovascular Interventions 
Heart House, 2400 N Street NW, Washington, DC, 20037

Ph: 202-375-6136

Fax: 202-375-6819

Email: jaccint@acc.org

Reviewer\#1 (Comments for the Author (Required)):

The authors have adequately answered my queries.

Reviewer \#2 (Comments for the Author (Required)):

1) Consider removing renal interventions entirely from the paper.

2) I do not understand why there was not an increase in tibial interventions, given the near doubling of interventions for CLI. Please add some comments to the discussion as to why this is this case.

3) For devices, does "balloon" include drug-coated balloons? While DCB may not have been available for many of the years included, they may have been used as part of trials in the latter years. Likewise, it is not clear to me how devices were counted- if intervention entailed balloon angioplasty followed by stent, does this make it into the data once as a "stent" procedure?

4) In discussion, consider adding more comments regarding why CLI intervention has increased. In addition to using endovascular techniques in place of surgery, there is probably better awareness and more rapid detection of CLI. Additionally, endo technologies make most CLI anatomy approachable in the modern area, and many of the procedures in your database may have been done for $\mathrm{CLI}$ restenosis (treating $\mathrm{CLI}$ leads to lots of surveillance, restenosis, and repeat procedures). 


\section{Reviewer's Comments}

\section{Reviewer \#1}

In the current manuscript, the authors investigate temporal trends in peripheral vascular interventions and device usage from 2006-2013 based on 43 hospitals participating in the Michigan BMC2 VIC registry. The study identified several trends, including decreased rates of renal artery stenting, increased treatment of femoropopliteal disease, and a greater proportion of patients with critical limb ischemia being treated. Device usage was also investigated, with trends for increased balloon angioplasty and less cryoplasty, but reportedly no change in stent rates, atherectomy, or laser. These temporal trends are important and add value to the existing data on procedural trends, but I have several questions related to the methodology and results:

We thank the reviewer for identifying the importance of the manuscript and hope to answer the questions below.

1. In the introduction, the statement that PVI offers a mortality benefit over surgery is overstated. While it is true that PVI is lower risk from an intervention standpoint acutely, no long-term outcomes have demonstrated a mortality benefit (and in fact the BASIL study suggested a benefit of surgery among patients who lived two years).

We agree with the reviewer that the comment is overstated and have changed the statement to read "Endovascular peripheral vascular interventions continue to increase and often offer advantages over surgical interventions".

2. Why were PVIs defined as renal and leg interventions? This seems rather arbitrary, because if renal interventions are included then carotid, subclavian and mesenteric interventions should probably be included. Otherwise the manuscript would be more focused if just focused on aorto-iliac, femoropopliteal, and below the knee disease.

We thank the reviewer for the comment and agree that we have only selected a subset of peripheral interventions. On examining the initial data from BMC2, upper extremity and mesenteric rates were low and therefore, excluded, while carotid interventions were not included. Although not related to the treatment of claudication or critical limb ischemia, we felt that including renal interventions was important as it highlights a change in practice patterns.

3. What is the documentation or criteria provided for claudication, rest pain, or arterial ulcer in the database? For example, in the absence of hemodynamic criteria, rest pain can be a subjective indication for intervention.

We thank the reviewer for the comment and agree that the definition may be subjective. To that end, we have included the definitions from the BMC2 PVI Registry that were used at that time and can place these in the Methods section if desired.

Claudication: leg pain caused by poor circulation, inhibiting patient's ability to walk distances. Refers to cramping pain in the legs (usually the calf muscles, but may be in 
the thigh muscles) caused by poor circulation of the blood in the arteries of the leg muscles during exercise. True claudication is relieved with rest from exercise.

Rest pain: the patient complains of ischemic symptoms in the legs while at rest or during the night, or if narcotics are used to relieve symptoms.

Ulcer present: If the patient has an ulcer or tissue loss is present.

4. The Results showing increased CLI interventions over time but no increase in below knee interventions seem discordant. If there were more CLI interventions, there should have also been more below knee interventions.

We thank the reviewer for the comment and would like to refer to Table 3 . This table shows that the proportion of below-the-knee interventions does increase over time from $15.6 \%$ in 2006 to $26.1 \%$ in 2013 . This is not statistically significant because there were so few hospitals in the earlier time points. Unfortunately, we can't overcome this with additional analysis. However, we do agree that it merits further discussion. Therefore, we have added to the Discussion: "As shown in Table 3, rates of aorto-iliac are relatively stable while the proportion of below-the-knee interventions does increase over time, but is not statistically significant. This may be secondary to the smaller number of hospitals in the Registry in the earlier time points as one would expect an increase in below-the-knee interventions with an increased proportion of procedures performed for critical limb ischemia.

5. It is hard to determine how a peripheral intervention would be performed without balloon angioplasty. How is this defined? As stand-alone balloon angioplasty?

We thank the reviewer for the comment. Balloon angioplasty is a stand-alone category meaning that patients with other interventions (stent, atherectomy, etc.), but who also had balloon angioplasty be classified into those other categories while patients classified as balloon angioplasty had balloon angioplasty alone. This information can be added to the manuscript if desired.

6. Laser is a type of atherectomy, so it seems odd that laser is separated out in the abstract, Methods and Results.

We thank the reviewer for the comment and agree that laser can be grouped with other debulking devices. However, it is separated in the BMC2 PVI Registry and we have therefore continued with this distinction.

7. Many peripheral vascular procedures necessitate the use of combined devices, e.g., atherectomy, balloon angioplasty, and then stenting. How was this accounted for in the analysis? It would be helpful as well to have more Results regarding combination of these therapies, especially in the femoropopliteal segment.

We thank the reviewer for the comment. As noted in \#5, each therapy is coded separately, except balloon angioplasty, so they can be combined any number of ways 
desired. We agree that it would be helpful to have results regarding combination of these therapies, however, there are over 5040 possible combinations.

8. Cryoballoons are available again on the market, so the discussion should be revised to reflect that fact.

We thank the reviewer for the comment and have changed the discussion to reflect this important point.

\section{Reviewer \#2}

Thomas et al. report the temporal trends in peripheral interventions (renal and leg) from a large Michigan registry during years 2006-2013. They identified that renal interventions declined significantly, while femoropopliteal procedures increased. Procedures for critical limb ischemia nearly doubled during this time period.

Please address the following comments/questions:

1) Does the BCBS registry include all PVI procedures or just those of patients with this private insurance?

We thank the reviewer for this question. The PVI Registry collects data on all patients at each hospital receiving PVI irrespective of their insurance status. This information can be included in the manuscript if desired.

2) How were multilevel procedures (eg simultaneous fempop plus below knee intervention for a patient with CLI) handled for the purposes of analysis? It is common for below-knee interventions to be performed concurrently with more proximal interventions.

We thank the reviewer for the comment. If the patient had multiple procedures (locations) performed on the same day or different hospital days during the same admission, they are recorded as separate procedures. We, too, are interested in different combinations of procedures (locations), but the number of combinations is too numerous for statistical analysis.

3) Do you have any data on interventions done for restenosis? I wonder if some of the trends, particularly those related to the femoropopliteal segment, reflect treatment of restenosis and not necessarily new patients.

We thank the reviewer for the comment. We do have that data within the database, but it was not analyzed for this current manuscript.

4) It is somewhat discordant to note that the CLI indication nearly doubled, but a significant increase in below-knee interventions was not detected. A large proportion of CLI patients get below-knee revascularization. Is this simply a power/sample size limitation? Is there any concern about the accuracy of the reporting within the registry-

\section{Catheterization and Cardiovascular Interventions}

This article is protected by copyright. All rights reserved. 
where leg interventions are being given CLI indications when really just claudication was present (eg operator gaming)?

We thank the reviewer for the comment and would like to refer to Table 3. This table shows that the proportion of below-the-knee interventions does increase over time from $15.6 \%$ in 2006 to $26.1 \%$ in 2013 . This is not statistically significant because there were so few hospitals in the earlier time points. Unfortunately, we can't overcome this with additional analysis. However, we do agree that it merits further discussion. Therefore, we have added to the Discussion: "As shown in Table 3, rates of aorto-iliac are relatively stable while the proportion of below-the-knee interventions does increase over time, but is not statistically significant. This may be secondary to the smaller number of hospitals in the Registry in the earlier time points as one would expect an increase in below-the-knee interventions with an increased proportion of procedures performed for critical limb ischemia." In regards to the accuracy of the reporting within the Registry, the data is audited by experienced staff and is accurate to the best of our knowledge.
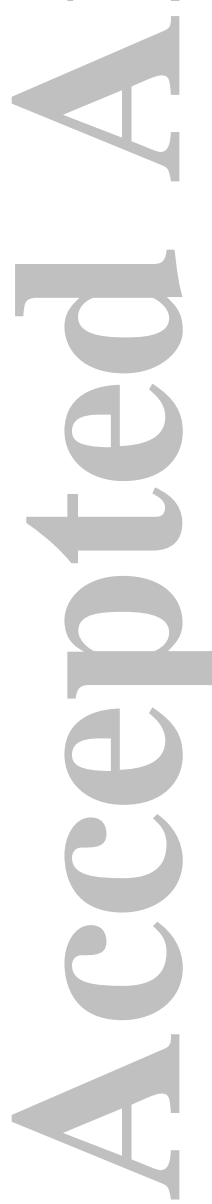


\title{
Temporal Trends in Peripheral Arterial Interventions: Observations from the Blue Cross Blue Shield of Michigan Cardiovascular Consortium (BMC2 PVI)
}

\section{Short Title: Trends in Peripheral Arterial Interventions}

Michael P. Thomas, MD, ${ }^{*} \dagger$ Yeo Jung Park, PhD, ${ }^{*}$ Scott Grey, PhD, ${ }^{*}$ Theodore L Schreiber, MD, $\ddagger$ Hitinder S Gurm, MD, ${ }^{*} \dagger$ Dale Leffler, DO,§ Thomas P Davis, MD, ॥ Peter Henke, MD, ${ }^{*}+\mathrm{P}$ Michael Grossman, $\mathrm{MD}^{*} \dagger$

*University of Michigan, Ann Arbor, MI; †VA Ann Arbor Healthcare System, Ann Arbor, MI; ‡Detroit Medical Center, Detroit, MI; §Sparrow Health System, Lansing, MI; ||Eastlake Cardiovascular, P.C., St Clair Shores, MI

Support: Support for BMC2-VIC is provided by Blue Cross and Blue Shield of Michigan and Blue Care Network as part of the BCBSM Value Partnerships program. The funding source was not involved in any part of this study. Disclaimer: Although

Blue Cross Blue Shield of Michigan and BMC2 work collaboratively, the opinions, beliefs, and viewpoints expressed by the author do not necessarily reflect the opinions, beliefs, and viewpoints of BCBSM or any of its employees.

\author{
$r$ \\ Key Words \\ Peripheral arterial disease \\ Peripheral vascular interventions \\ Temporal trends \\ Claudication \\ Critical limb ischemia \\ Device usage \\ Word Count: 3005 \\ Corresponding Author: \\ Michael P. Thomas, MD \\ The University of Michigan \\ Cardiovascular Medicine \\ Cardiovascular Center \\ 1500 E. Medical Center Dr., SPC 5869 \\ Ann Arbor, MI 48109-5869 \\ 734-615-3878 (phone) \\ 734-764-4142 (fax) \\ michptho@med.umich.edu
}




\section{Structured Abstract}

\section{Objectives}

The aim is to examine trends in procedural indication, arterial beds treated, and device usage in peripheral arterial interventions.

\section{Background}

There is little data on indication, vascular beds treated and devices utilized for peripheral arterial interventions (PVIs).

\section{Methods}

We used data from 43 hospitals participating in the BMC2 VIC registry. PVIs were separated by year and divided by arterial segment. Lower extremity PVIs were subclassified as having been performed for claudication or critical limb ischemia.

Yearly device usage was also included. A repeated measure ANOVA was used to determine trends.

\section{Results}

44,650 PVIs were performed from 2006 to 2013 . Renal interventions decreased from $18 \%$ of interventions in 2006 to $5.6 \%$ in $2013(\mathrm{p}<0.001)$ and femoralpopliteal increased from $54.9 \%$ in 2006 to $64.5 \%$ in 2013 ( $p<0.001$ ). No significant trend was seen for aorta-iliac or below-the-knee interventions. $58.6 \%$ of PVIs were performed for claudication in 2006 and this decreased to 44.6\% in 2013 ( $p=0.025$ ). Indications for critical limb ischemia were $24.1 \%$ in 2006 and 47.5\% in 2013 (p < 0.001). There were significant increases in the use of balloon angioplasty ( $\mathrm{p}=$ 0.029) and cutting / scoring balloons ( $<<0.001)$ while cryoballoon usage decreased 
( $p<0.001)$. No significant changes were found with stenting, atherectomy, and laser.

\section{Conclusions}

There is a significant increase in patients presenting with critical limb ischemia.

Renal artery intervention rates are decreasing while femoral-popliteal interventions are increasing. Additionally, balloon angioplasty and cutting / scoring balloon usage (1) is increasing.



\section{Abbreviations}

$\mathrm{PAD}=$ peripheral arterial disease

$\mathrm{PVI}=$ peripheral vascular interventions

BMC2 PVI = Blue Cross Blue Shield of Michigan Cardiovascular Consortium

Peripheral Vascular Intervention Registry
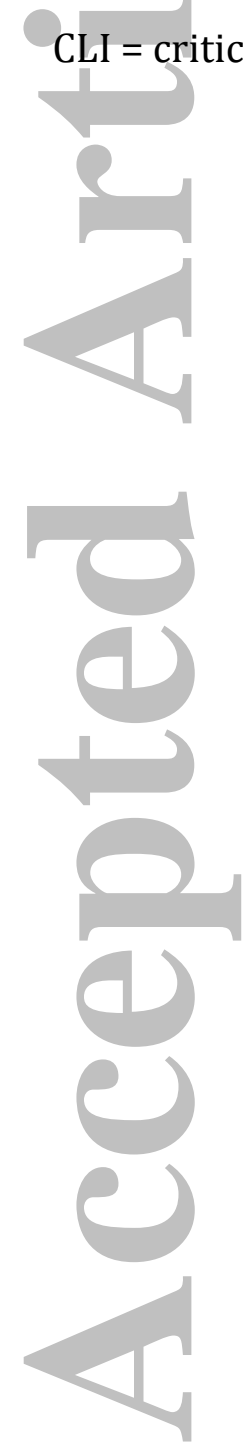


\section{Introduction}

Peripheral arterial disease (PAD) affects approximately 8.5 million Americans $>/=40$ years of age. PAD is associated with significant morbidity and mortality, potential limb loss, and a negative impact on quality of life. The manifestations of PAD are broad and range from asymptomatic to intermittent claudication to critical limb ischemia with tissue loss. (1) In recent years, multisociety efforts have resulted in guidelines for PAD management. (2) Endovascular peripheral vascular interventions (PVI) continue to increase and often offer advantages over surgical intervention. (3) PVIs have become an important part of practice of interventional cardiologists, interventional radiologists and an increasingly utilized approach for vascular surgeons. Additionally, the PVI device market continues to grow. However, there are little contemporary data on the treatment patterns in PVI. It is in this setting that we sought to examine trends in current PVI practice including indication, vascular beds treated, and devices utilized.

\section{Methods}

$\underline{\text { Study Population }}$

The study population consisted of consecutive PVIs (renal, aorto-iliac, femoral-popliteal, and below-the-knee) from 1/2006 to 12/2013 in the Blue Cross Blue Shield of Michigan Cardiovascular Consortium Peripheral Vascular Intervention (BMC2 PVI) Registry. BMC2 PVI Registry 
The details of the BMC2 PVI Registry have been described previously. (4) Briefly, BMC2 PVI is a prospective, multicenter, observational quality improvement registry funded by Blue Cross Blue Shield of Michigan. The registry is a regional collaborative effort aimed to improve the quality of care and patient outcomes and overcome barriers of traditional market and academic competition. The registry collects, audits, and organizes data and reports procedural variables and outcomes to individual operators and institutions. A data form is compiled for each patient, including demographic information, past medical history, laboratories pre- and post-PVI, patient history, presenting symptoms, procedural indications, medication details, PVI types, details of procedure, and associated complications if present. Data quality is ensured by ad hoc queries, random chart reviews, and a series of diagnostic routines included in the database. Peri-procedural and in-hospital data are collected from each individual. The registry has been approved or the need for approval waived by the institutional review board of each participating hospital.

\section{Statistical Analysis}

\section{Location and Indication}

Consecutive PVIs from 1/2006 to 12/2013 were separated by year and were divided by arterial segment (renal, aorto-iliac, femoral-popliteal or below-the-knee) The lower extremity PVIs were sub classified as having been performed secondary to either claudication or critical limb ischemia (CLI) defined as rest pain or ulcer. In order to determine changes in temporal trends, the p-value was calculated using a repeated measure ANOVA.

Device Usage 
PVI devices included balloon, stent, atherectomy, cryoballoon, cutting balloon, and laser. A repeated measure ANOVA was used to determine temporal trends in device usage.

\section{Sensitivity Analysis}

The analysis was repeated utilizing only the original 6 hospitals in the registry from 2006-2013 to ensure that the changes observed were not secondary to a difference in treatment culture within the new centers.

\section{Results}

A total of 44,650 consecutive patients undergoing PVI were prospectively enrolled from 43 hospitals in Michigan state from January 2006 through December 2013 (Table I). Baseline demographic and clinical characteristics are detailed in

Table II. Across any given year, approximately $41-45 \%$ of the patients were female and the cohort had an average age of 68 years-old with a BMI ranging from $28-32$ $\mathrm{kg} / \mathrm{m}^{2}$. Nearly one-third of patients were current smokers and $43.5 \%-58.5 \%$ had a history of smoking. Nearly one-half of patients had a history of diabetes and approximately two-thirds had concomitant coronary artery disease. Additionally, over $80 \%$ of patients had a history of hyperlipidemia while over $90 \%$ had a history of hypertension.

A significant temporal trend was observed for procedural indication from 2006 to 2013 . In $2006,58.6 \%$ of the 1677 PVIs were performed for claudication while $44.6 \%$ of the 9185 PVIs were performed for claudication in 2013 ( $p=0.025)$. 
Critical limb ischemia comprised $24.1 \%$ of the interventions in 2006 and increased to $47.5 \%$ of the PVIs in $2013(\mathrm{p}<0.001)$. (Figure 1)

There were also significant changes in the location of the vascular beds that were treated over the study period. Renal interventions accounted for $18 \%$ of the overall PVIs in 2006 and decreased to $5.6 \%$ of the interventions in $2013(\mathrm{p}<0.001)$.

Femoral-popliteal interventions accounted for 54.9\% of PVIs in 2006 and increased to comprise $64.5 \%$ of the interventions in $2013(\mathrm{p}<0.001)$. No significant trend was seen in the percent of overall cases comprised of aorta-iliac or below-the-knee interventions from 2006 to 2013 (Table III) In 2006, 67.6\% of PVIs utilized balloon angioplasty and this increased to $85.4 \%$ in $2013(p=0.029)$. Cryoballoon usage decreased from $5 \%$ in 2006 to $0.6 \%$ in 2013 ( $p<0.001$ ) while cutting/scoring balloon use increased from $1.8 \%$ in 2006 to $15.1 \%$ in 2013 ( $p<0.001)$. There was no significant temporal trend found in the use of stenting, atherectomy, and laser from 2006 to 2013. (Table IV)

\section{Sensitivity Analysis}

A total of 13,383 consecutive PVIs were performed at the original 6 hospitals in the registry from 2006 through 2013 (Table I). A significant temporal trend was observed for procedural indication as claudication decreased $(\mathrm{p}=0.046)$ and critical limb ischemia increased $(\mathrm{p}<0.001)$. (Supplementary Figure 1) In regards to vascular beds treated, there was a significant decrease in renal artery interventions and increase in femoral-popliteal and below-the-knee interventions while there was no significant trend observed in aorto-iliac interventions. (Supplementary Table I) There were significant increases observed in the use of balloon and cutting 
/scoring balloons while there were significant decreases seen in stenting, cryoballoon and laser therapy. There was no significant trend observed with the use of atherectomy. (Supplementary Table II)

\section{Discussion}

In this large cohort of PVI patients from 2006 to 2013, we observed several changes in indication for the procedure, vascular bed treated and device utilization over the study period. In more recent years, there have been a greater proportion of patients being treated for critical limb ischemia. The rates of renal artery interventions decreased over time, while the rates of femoral-popliteal artery interventions increased. Additionally, balloon angioplasty and cutting balloon usage increased, while cryoballoon therapy declined. Atherosclerotic risk factors were widely prevalent in this cohort and there were no major changes in the patient r. baseline characteristics.

\section{Procedural Indication}

The key finding of our study is that there has been a significant shift in the type of procedures that are being performed. Over the study period, there was an increase in the PVI procedural indication of critical limb ischemia and decrease in claudication from 2006 through 2013. In 2006, approximately one in four cases (24.1\%) were completed for CLI while this increased to nearly one-half in 2013 $(47.5 \%)$

It is likely that the increased prevalence of CLI as an indication in our study is a reflection of the increasing recognition of the safety and efficacy of percutaneous 
versus surgical revascularization in patients with significant co-morbidities. Nasr et al. reviewed single center institutional revascularization rates for critical limb ischemia from 1994 to 1999 . They found that PTA rates increased from $44 \%$ in 1994-1995 to 69\% in 1998-1999 while surgical rates declined. There was no statistically significant difference between the groups in regards to patient survival, limb salvage rates, and mean hospital stay. (5) Plaisance et al. examined the safety of PVI in the elderly in a statewide registry consisting of 7,769 patients. The authors found that the elderly patients presented with more severe PAD including rest pain or goal of limb salvage, however, after adjustment for baseline covariates, advanced age was not associated with increased rates of MACE, transfusion, contrast induced nephropathy, or amputation. (6) Furthermore, surgical revascularization for critical limb ischemia has been found to be associated with a higher 30-day and 1year mortality in octogenarians when compared to younger patients. (7) Also, Faglia et al. found that percutaneous revascularization was feasible in $84 \%$ of diabetic patients presenting with CLI as a first choice revascularization procedure and had an associated low complication rate (3.4\%). (8) Therefore, it is not surprising that there is increased utilization of PVI for CLI patients..

\section{Arterial Bed Treated}

Renal artery interventions decreased from 2006 to 2013 while rates of

femoral-popliteal increased. There was no significant trend observed in rates of aorto-iliac or below-the-knee interventions during this time period. As shown in Table III, rates of aorto-iliac are relatively stable while the proportion of below-theknee interventions does increase over time, but is not statistically significant. This 
may be secondary to the smaller number of hospitals in the Registry in the earlier time points as one would expect an increase in the below-the-knee interventions with an increased proportion of procedures performed for critical limb ischemia. In our study, the rate of renal artery intervention dropped from 18\% in 2006 to approximately $6 \%$ in 2010 . This percentage remained relatively stable through 2013. Notably, this decrease in the rate of renal artery intervention was prior to the publication of the CORAL study in 2014, which confirmed that effective medical therapy should be the first line of treatment in patients with presumed renovascular hypertension. (9) It is likely that the widespread uncertainty and the clinical equipoise about the efficacy of renal stenting resulted in a decline of this procedure even prior to the publication of the CORAL trial.

Goodney et al. examined trends in lower extremity revascularization practices in Medicare beneficiaries from 1996 to 2006 and found that endovascular treatment increased three-fold during this time period while lower-extremity bypass decreased by $42 \%$. (10) This change in practice may have been partially driven by patient preference for less invasive procedures that paralleled advances in catheter-based technologies. This practice pattern is consistent with our observation of an increase in the rate in femoral-popliteal interventions from 2006 to 2013 .

Device Utilization

Data from our large registry would suggest that plain balloon angioplasty and cutting/scoring balloon angioplasty has increased while cryoballoon therapy is decreased. In fact, the cryoballoon was withdrawn from the U.S. market for a period 
of time and recently reintroduced. Therefore, these rates may begin to increase again. Additionally, there was no significant trend identified in the use of stents, atherectomy devices, or laser from 2006 to 2013. The ACC/AHA guidelines for the management of patients with PAD provide a basic framework for utilization of these devices. (11) Stenting for iliac artery stenosis is a Class I LOE B recommendation while stenting and other adjunct therapies can be useful for salvage therapy from balloon dilatation of femoral, popliteal, or tibial vessels (Class IIb LOE C recommendation).

Along with these basic guidelines, the device market for treatment of PAD continues to expand rapidly and adjunct therapies are more readily available including drug eluting balloons and atherectomy devices. Given the number and variability in treatment modalities, as well as practitioner-preference and the lack of significant clinical trial data, the interventionalist or surgeon has a wide array of devices to chose from in treating in a specific lesion.

To our knowledge, this is the largest study to examine changes in device usage in treatment of peripheral arterial disease.

\section{$\underline{\text { Limitations }}$}

The results in this study are based upon observational data that are not adjudicated by a central facility. The use of a large registry with clinical heterogeneity and reporting variability leads to the possibility for confounding variables and treatment bias. Additionally, all patients in our study underwent PVI at hospitals participating in a quality improvement initiative and therefore, these findings may not apply to other institutions or patients from other areas. However, the data is based on a 
large registry and reflects continuous data on unselected patients undergoing PVI procedures performed by multiple specialists from different backgrounds (interventional cardiology, interventional radiology, vascular surgery) and offers a contemporary insight into treatment in this population.

\section{Conclusions}

In this study, a there was a significant increase in the proportion of patients that presented for lower extremity PVI with critical limb ischemia, while fewer presented with claudication. From 2006-2013, the rates of renal artery interventions decreased while femoral-popliteal interventions increased.

Additionally, the use of balloon angioplasty and cutting/scoring balloon increased from 2006 to 2013. These findings shed light on contemporary practice patterns related to the percutaneous management of PAD and suggest the need for additional studie studies to define optimal treatment strategies.

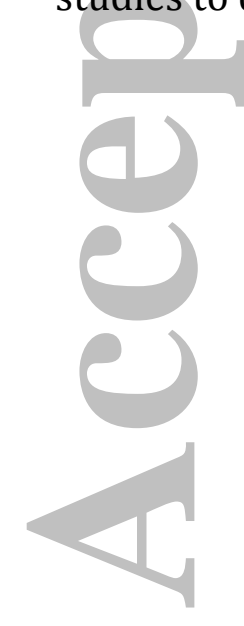




\section{References}

1. Go AS, Mozaffarian D, Roger VL et al. Heart disease and stroke statistics-2014 update: a report from the American Heart Association. Circulation 2014;129:e28-e292.

2. Hirsch AT, Haskal ZJ, Hertzer NR et al. ACC/AHA 2005 guidelines for the management of patients with peripheral arterial disease (lower extremity, renal, mesenteric, and abdominal aortic): executive summary a collaborative report from the American Association for Vascular Surgery/Society for Vascular Surgery, Society for Cardiovascular Angiography and Interventions, Society for Vascular Medicine and Biology, Society of Interventional

Radiology, and the ACC/AHA Task Force on Practice Guidelines (Writing

Committee to Develop Guidelines for the Management of Patients With Peripheral Arterial Disease) endorsed by the American Association of Cardiovascular and Pulmonary Rehabilitation; National Heart, Lung, and Blood Institute; Society for Vascular Nursing; TransAtlantic Inter-Society Consensus; and Vascular Disease Foundation. Journal of the American College of Cardiology 2006;47:1239-312.

3. Nowygrod R, Egorova N, Greco G et al. Trends, complications, and mortality in peripheral vascular surgery. Journal of vascular surgery 2006;43:205-16.

4. Mukherjee D, Munir K, Hirsch AT et al. Development of a multicenter peripheral arterial interventional database: the PVD-QI2. American heart journal 2005;149:1003-8. 
5. Nasr MK, McCarthy RJ, Hardman J, Chalmers A, Horrocks M. The increasing role of percutaneous transluminal angioplasty in the primary management of critical limb ischaemia. European journal of vascular and endovascular surgery : the official journal of the European Society for Vascular Surgery 2002;23:398-403.

6. Plaisance BR, Munir K, Share DA et al. Safety of contemporary percutaneous peripheral arterial interventions in the elderly insights from the BMC2 PVI (Blue Cross Blue Shield of Michigan Cardiovascular Consortium Peripheral Vascular Intervention) registry. JACC Cardiovascular interventions 2011;4:694-701.

7. Brosi P, Dick F, Do DD, Schmidli J, Baumgartner I, Diehm N. Revascularization for chronic critical lower limb ischemia in octogenarians is worthwhile. Journal of vascular surgery 2007;46:1198-207.

8. Faglia E, Dalla Paola L, Clerici G et al. Peripheral angioplasty as the firstchoice revascularization procedure in diabetic patients with critical limb ischemia: prospective study of 993 consecutive patients hospitalized and followed between 1999 and 2003. European journal of vascular and endovascular surgery : the official journal of the European Society for Vascular Surgery 2005;29:620-7.

9. Cooper CJ, Murphy TP, Cutlip DE et al. Stenting and medical therapy for atherosclerotic renal-artery stenosis. The New England journal of medicine 2014;370:13-22. 
10. Goodney PP, Beck AW, Nagle J, Welch HG, Zwolak RM. National trends in lower extremity bypass surgery, endovascular interventions, and major amputations. Journal of vascular surgery 2009;50:54-60.

11. Anderson JL, Halperin JL, Albert NM et al. Management of patients with peripheral artery disease (compilation of 2005 and 2011 ACCF/AHA

guideline recommendations): a report of the American College of Cardiology Foundation/American Heart Association Task Force on Practice Guidelines. Circulation 2013;127:1425-43.

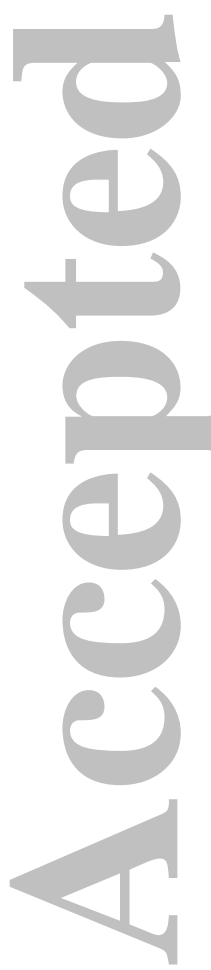




\section{Figure Legend}

Figure 1 - Procedural Indication

Shown is the temporal changes in the proportion of patients undergoing peripheral vascular intervention for claudication and critical limb ischemia.
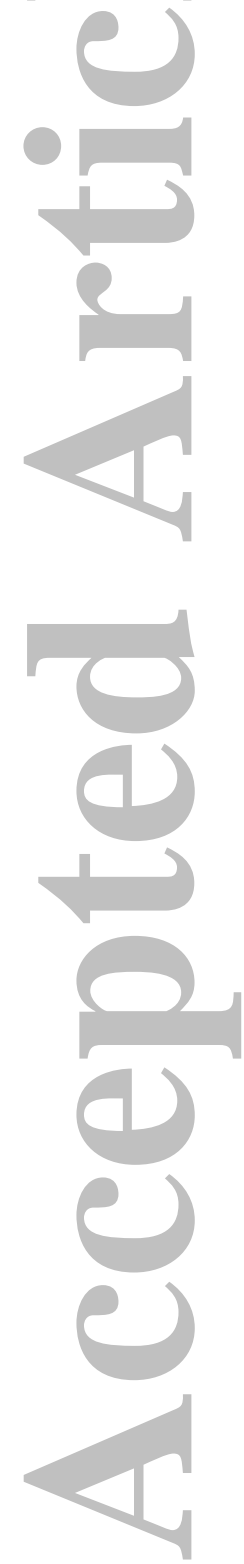